This is an Accepted Manuscript of an article to be published by Taylor \& Francis in Building Research and Information, available online: http://dx.doi.org/10.1080/09613218.2016.1212299

Energy-saving occupant behaviours in offices: change strategies.

\author{
Mark Mulville* \\ University of Greenwich \\ E-mail: $\underline{\text { m.mulville@greenwich.ac.uk }}$ \\ Keith Jones \\ Anglia Ruskin University \\ keith.jones@anglia.ac.uk \\ Gesche Huebner \\ University College London \\ g.huebner@ucl.ac.uk \\ Joanne Powell-Greig \\ Edge Hill University \\ joanne.powell@edgehill.ac.uk
}

*Author for correspondence 


\title{
Energy-saving occupant behaviours in offices: change strategies.
}

\begin{abstract}
As regulated energy consumption in buildings is reduced; the proportional importance of unregulated energy consumption increases. Reducing unregulated energy use in the commercial office requires an understanding of the factors that influence workplace behaviour. To date these factors have been assumed to be similar to those that influence behaviour in the home. However, the social dynamics of the workplace are different to that in the home. This study examined the degree to which theories of behaviour change generated largely in a domestic building setting could be used as the basis for designing interventions to reduce unregulated energy consumption in the workplace. The study examined the unregulated energy consumption of 39 workers engaged in office type activities in two separate locations. Following a 100 day monitoring period, three behaviour change interventions were developed and their impact measured over a 100 day period. Results from the study found, on average, an $18.8 \%$ reduction in energy use was achieved. Furthermore, by comparing pre and post intervention responses to an environmental questionnaire it was evident that savings were realised without significant changes to pro-environmental attitude or perceived social norms, which may have implications for energy saving interventions in the commercial sector.
\end{abstract}

Keywords: Behaviour change, building management, energy, feedback, social norms 


\section{Introduction}

The European Commission's 2030 climate and energy framework (2014) has targeted a 27\% increase in energy efficiency to support a low carbon economy and achieve sustainable growth. In 2011 nonresidential buildings (EU, Switzerland and Norway) consumed approximately 146 Mtoe (million tonnes of oil equivalent) of fuel, this represents $32 \%$ of the total consumption across all buildings, but for only 25\% of the total building stock (Building Performance Institute Europe, 2011). If the EU is to meet its 2030 targets, non-residential buildings must reduce their energy consumption.

Changes to the built environment regulatory framework (such as Part L of the UK Building Regulations) aim to reduce the heating, cooling and lighting (regulated) energy consumption in new buildings. Additionally, environmental/energy assessment methods and standards such as Passive House, BREEAM and LEED should encourage designers and developers to go beyond the minimum standards required by regulations. However, as these savings are predominantly technical interventions targeted at new buildings, and due to the legacy of the existing stock their impact on overall energy consumption will take considerable time to realise. In comparison, reductions in unregulated energy are largely unaffected by legacy design and as such, impacts here could be realised much more quickly. However, it is unclear what approaches could best be used to reduce unregulated energy in commercial buildings.

Unregulated energy use in non-domestic buildings is that energy associated with small power devices, office equipment, desktop and laptop computers and localised heating, cooling and lighting. Although the energy efficiency of individual pieces of equipment is likely to have improved in recent years (Mulville, Jones \& Huebner, 2014), the increased range of equipment used has led to predictions that small power will continue to have a significant impact on overall energy use in the future (Jenkins, Singh, \& Eames, 2009). Energy used at individual workstations may account for up to $88 \%$ of total office equipment energy use (Junnila, 2007). At the same time, actual utilisation of equipment by occupants, while the equipment is powered on, may be as low as $43 \%$ (Kawamoto, Shimoda \& Mizuno, 
2003), indicating that large savings may be possible and that occupant behaviour could have a significant impact on this (Zhang, Siebers \& Aickelin, 2011). Arguably, many campaigns to reduce energy use in the workplace are based on information provision only, driven by corporate social responsibility (CSR). However such a passive approach, as noted by Carrico and Riemer (2011), may not be enough for significant savings to be achieved.

Until recently most studies that have examined energy saving behaviour have done so in a domestic setting (for a review see Abrahamse, Steg, Charles \& Rothengatter, 2005) taking an individualistic approach to energy saving behaviour and suggesting the individual's beliefs and attitudes towards pro-environmental behaviour are paramount. However, whilst an individual's approach, beliefs and attitudes towards pro-environmental behaviour may be transferable to the workplace, it is likely that organisational factors (culture, organisational focus and structure) (Tudor, Barr \& Gilg, 2008) and job satisfaction (Brent and Freathy, 1997) will influence their importance. In the workplace the disconnect between the energy user and who pays the bills, the high density of occupants and perceived lack of control may also be influential as users feel disconnected from the space they occupy (Bull, Lemon, Fleming, Stuart \& Everitt, 2014) . This therefore calls into question the applicability of theories adopted from residential sector to energy saving behaviour within the commercial sector (Siero, Bakkerm, Dekker \& van Der Burg, 1996; Carrico \& Riemer, 2011 cited in Nilsson, Andersson \& Bergstad, 2015 and Chen \& Ahn, 2014). Application of energy saving behaviour theories in the commercial office setting have to date been limited to a small but growing number of studies (for example Tetlow, Beaman, Elmualim \& Couling, 2013; Gulbinas \& Taylor, 2014; Murtagh et al., 2013). This study aims to build on those preceding studies and examines the effectiveness of behaviour change interventions using feedback, goal setting and social normative influence on energy consumption within commercial office buildings. 


\section{Theoretical Framework}

As noted by Chatterton (2011) there has been significant developments in our understanding of behaviour since the 1960s. As a result, a range of theories have emerged to help us explore and potentially influence behaviour. More recently, a range of approaches have been developed in relation to energy use behaviour particularly in domestic buildings (for a review see Chatterton, 2011). These approaches include economic, psychological, sociological and educational theories and when considered in the context of a behavioural model it may be possible to use them to influence behaviour (Chatterton, 2011). There is a wide range of models of behaviour, they often overlap or build upon one another and can be seen as complimentary. Triandis' "Theory of Interpersonal Behaviour" has been shown to be useful in relation to energy use behaviour (Chatterton, 2011) and is used here (see Figure 1) to highlight the interrelationships between the factors discussed in the remainder of this review.

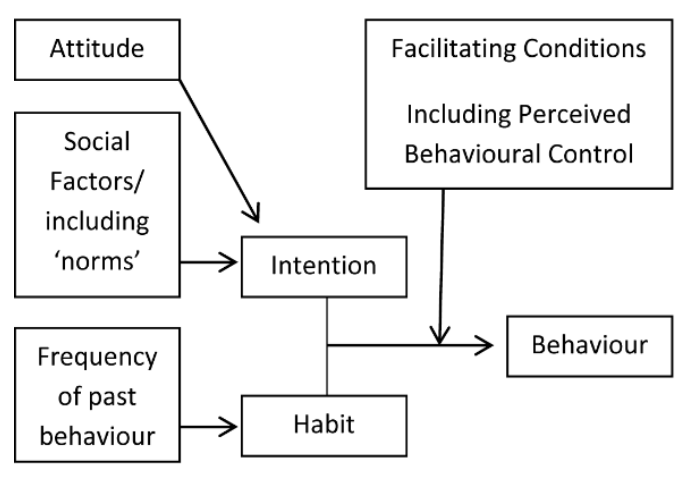

Figure 1: Simplified version of Triandis" "Theory of Interpersonal Behaviour", adapted from Chatterton, 2011 
Habit, Attitude and Intention

Chatterton (2011) argued that intentions and behaviour, over time, form habit or 'locked-in' practice (Maréchal, 2010) which is difficult to change. In the short term it may be possible to change behaviour through conscious effort but unless this becomes habitual the change is unlikely to persist. Maréchal (2010) suggests that habit, as a barrier to change, is unconscious (and as such is automatic) while conscious decisions occur rarely, habits are embedded in everyday routines. The time taken for a habit to become automatic (as defined by Bargh, 1994 in Chatterton, 2011) is reported to be approximately 66 days (Lally, van Jaarsveld, Potts \& Wardle, 2010). Where a given issue (such as climate change) is perceived to be beyond the individual's immediate self-interest changing a habit may be more challenging (Stern \& Gardener, 1981). Francis et al. (2004) noted strong links between attitude, intention and behaviour. A change in behaviour may require a change in values and/or attitude, however there is some evidence that this is not always the case, DeYoung (1995) for instance, found that recyclers and non-recyclers did not differ in their attitudes toward recycling.

\section{Social Norms}

Social norms are those attitudes or behaviours that are considered to be the 'norm' within the group that the individual belongs to i.e. it is what the majority of our peers say, think do etc.. Social norms can be particularly influential where people seek to be praised or rewarded by others (Chatterton, 2011). A group's social norms are usually an implicit set of rules for acceptable behaviours, values and beliefs (Miller \& Prentice, 1996) within the group. Those who do not conform are perceived to be different, difficult and a general hindrance to maintaining social order. A realisation of a mismatch between one's own behaviour and social norms may lead to feelings of guilt (Baumeister, 1998 in Gilbert, Fiske \& Lindzey, 1998).

Normative social influence has been explored in relation to pro-environmental behaviours (Hopper \& Neilson, 1991; Nolan, Schultz, Cialdini, Goldstien \& Griskevicius, 2008; Scherbaum, Popovich \& Finnlinson, 2008). Nolan et al., (2008) explored the extent to which norms influenced energy 
conservation behaviours. They found that individuals who had been given a descriptive normative message containing information about the conservation behaviours of the majority of others made significant energy savings. The study demonstrated that social norms, whether true or not can actually motivate people to conserve energy. Scherbaum, et al. (2008) in a study Influenced by value-beliefnorm (VBN) theory (Stern, 2000; Stern, Dietz, Abel, Guagnano, \& Kalof, 1999), suggests that environmental personal norms and environmental worldviews can be leveraged by organisations in their interventions to reduce employee energy use.

Some studies have shown that communicating social norms can actually increase an undesirable behaviour (e.g. Perkins, Haines \& Rice, 2005; Wechsler et al., 2003; Werch et al., 2000). Schultz, Nolan, Cialdini, Goldstein and Griskevicius (2007) provided a descriptive normative message to 290 residents detailing energy consumption, households who were low-energy consumers, once given the message increased their energy use. This is because individuals use their perceptions of peer norms as a standard against which to compare their own behaviour. However, Schultz et al. (2007) also found that when an injunctive norm (the perception that the behaviour is commonly approved or disapproved within the group) was combined with the descriptive normative message low-energy users continued to consume energy at the desirable amount.

\section{Perceived Behavioural Control}

Perceived behavioural control as noted by Francis et al. (2004) is made up of two components, the persons control over the given or encouraged behaviour, such as the presence of suitable environmental controls in an office environment, and the persons confidence that they will be able to perform the behaviour, for instance successfully interacting with the given environmental controls. Perceived behavioural control is closely related to the presence of 'facilitating conditions' (Chatterton, 2011 in relation to Triandis 'Theory of Interpersonal Behaviour') which can make an encouraged behaviour easy or difficult, the knowledge of which may influence attitude and thus behaviour. 
Perceived behavioural control is a key element of the Theory of Planned Behaviour (with social norms and attitude) as developed by Ajzen (1991) and there is evidence that it can be influential in the success of energy saving measures in the commercial building sector. Menzes et al., (2012) in a study considering the turning off of lights and small appliances in the office setting, found a statistically significant relationship between perceived behavioural control and energy consumption, with those with higher perceived control saving more energy. In addition the same study found the impact of both social norms and attitude not to be significant, further highlighting the importance of perceived control. Bull et al. (2014) note however, that non-domestic building users can often feel detached or disconnected from the spaces they occupy which can be linked back to perceived control, this presents a particular challenge for building managers.

\section{Feedback}

Feedback can be a big motivator for encouraging sustainable behaviours (Bostrom \& Fischhoff, 2001). Both individual and group level feedback has been shown to positively influence pro-environmental behaviour (Dwyer, Leeming, Cobern, Porter \& Jackson, 1993; Schultz, Oskamp \& Mainieri, 1995). For feedback to be most effective it should be presented as close as possible in time and space to the behaviour that is being promoted and in such a way that it is simple to interpret (Benders, Kok, Moll, Wiersma \& Noorman, 2006; Carrico \& Riemer, 2011; Katzev \& Mishima, 1992; Siero et al., 1996), so that individuals can identify the relationship between their behaviour and the feedback (Seligman, Becker \& Darley, 1981). The effectiveness of feedback in the residential sector is shown to diminish once feedback is removed suggesting that feedback in order to be effective must be maintained in the long term (Dwyer et al., 1993). However, studies in commercial settings (Gulbinas \& Taylor, 2014; Murtagh et al., 2013) have found that although engagement with feedback reduces over time, energy savings may not occur until later in the study (when engagement with feedback has reduced). This perhaps suggests the commercial sector is less 'elastic' (Murtagh et al., 2013) than the residential in terms of the impact of feedback, however in some situations the non-domestic sector has been shown to be more elastic (such as Wakiyama, Zusman \& Monogan, 2014). Darby et al., (2016) (in an office 
environment within a higher education institution) found that when the level of feedback communicated (designed to reduced energy use) reduced there was a corresponding increase in energy consumption, suggesting that even where the response may be less elastic, continuous behavioural reinforcement may still be required to ensure persistence.

Comparative feedback, where the energy consumption of an individual or group is compared to the average, has been successful in reducing energy consumption in households, (Abrahamse et al., 2005). Comparative feedback works by evoking a feeling of competition via social comparison, or social pressure which then leads to changed behaviour in order to either correspond more closely to the 'norm' or 'perform better' than the group or other individual. This normative information can be provided by allowing individuals to believe that their performance will be compared with that of the other group (Shalley, Oldham \& Porac, 1987; Jackson \& Zedeck, 1982). Appraisal of the behaviour relative to the average consumption (or other group) can be given in the form of a positive (smiley/happy) or negative (unhappy) face. This phenomenon is explained by two different processes: social identity, where people will generally strive for a positive self-image (Tajfel, 1978) and competition.

Some promising research in commercial settings has demonstrated the positive effect of comparative feedback on reducing energy consumption (e.g. Siero, Boon, Kok \& Siero, 1989; Siero et al., 1996, Gulbinas \& Taylor, 2014). Siero et al. (1996) examined the effects of both group feedback (energy use of the group) and comparative feedback (energy use of other groups) in two units of a metallurgical company. Employees in the comparative feedback condition saved more energy than those who only received information about their own performance, even half a year after the intervention. Additionally, behaviour change took place with hardly any changes in attitudes or intentions. Gulbinas and Taylor (2014) in a study designed to understand the impact of feedback in a commercial office setting (where both comparative and individual feedback was used) found those that received individual feedback engaged less with the feedback and saved less energy than the comparative group. 
There may however be negative consequences to comparative feedback as people tend to avoid comparisons with others who perform better (Dakin \& Arrowood, 1981; van Knippenberg, Wilke \& de Vries, 1981).

\section{Goal Setting}

Van Houwelingen and Van Raaij (1989) note that goal setting offers motivation by setting out a desired future situation, achieving the desired outcome offers satisfaction which may also aid persistence through self-motivation. Reviewing preceding work in the area the authors go on to note that goals should be challenging yet achievable if savings of significance are to be realised. If the goal is too difficult and participants see no progress they are like to disengage, while goals that are too easy to achieve do not present the same rewards as more challenging ones. Abrahamse, Steg, Charles and Rothengatter (2007) expand on these ideas noting the potential benefits of goal-setting when combined with feedback, which enables participants to understand their progress while working towards the prescribed goal.

\section{Rationale for Interventions}

Based on the findings of the preceding review, for this study it was decided to combine feedback through a descriptive normative message (Cialdini et al., 1991), to allow the participant to understand the impact of their behaviours (Katzev \& Mishima, 1992) and goal setting (as per the findings of Abrahamse et al., 2007) with educational information (including information on how to save energy) to modify personal norms by appealing to the employees sense of environmental responsibility (Scherbaum et al., 2008). Darby (2006) notes in a review of the effectiveness of feedback on energy conservation that savings in the region of $20 \%$ may be possible and this was adopted for this study.

Taking this approach (feedback, goal setting and education) three groups were devised to further explore the effectiveness of (i) basic group feedback (to test the impact of feelings of group 
membership and social normative influence on energy savings), (ii) detailed group level comparative feedback (to build upon that of the first group and test the impact of feelings of competition) and (iii) individual and basic group feedback (to allow for comparison to the other groupings and understand the impact of social normative influence and feelings of guilt) in the commercial office setting.

To avoid a push towards the mean for those already using low amounts of energy (as highlighted by Shultz et al., 2007) the descriptive normative information in the form of regular feedback on energy use was combined with an injunctive normative information (supported by Schultz et al., 2007) in the form of a positive (smiley/happy) or negative (unhappy) face.

\section{Methods}

\section{Energy Consumption Monitoring and Procedure}

The energy use of 90 desks in two office buildings (within the same company) were monitored using 'Enistic' energy monitoring devices. These devices resemble an extension lead with four plug points and wirelessly logged energy readings on an hourly basis which was stored on a web accessible server. The devices were installed during an unoccupied period at each location and placed in a hidden cable tray under each desk. All desk top equipment was then routed through the Enistic device (screens, computer, laptop docking station etc.) including two desk top plugs made available for ad-hoc small power (fans, phone chargers and other small electronic devices).

Following installation a 100 Day 'baseline' period was established. This baseline period is longer than that used in other similar studies (such as Nilsson et al., (2015) and Gulbinas \& Taylor (2014)) and was intended to increase the reliability of the data by ensuring it was not heavily influenced by short periods of high or low energy use, which could be otherwise interpreted as energy savings (or increases). The consequence of this longer baseline period is a higher dropout rate, the implications of which are discussed below. The data gathered during the baseline period was analysed to establish baseline energy consumption for each participant. Corrections were made to allow for variations in 
working hours and staff absence. This was achieved through the establishment of out of hours power densities (the average power density at the desk location when not in use, which was taken as midnight on each day during the baseline period) and operational power densities (taken as the average during the working day). The data was then interrogated (every two weeks during the baseline and follow up monitoring period) to identify prolonged periods of low power densities indicating equipment was in sleep mode or had been powered down for an extended period, which was then used to establish the absence of the participants. The same approach (operational and out of hours power densities) was used to establish average working hours for each participant, which then allowed for the correction of the energy consumption data to ensure those who worked longer hours (or those who had been on leave) were not misrepresented, with the same working hours applied across all groups.

Following the completion of the baseline period a series of field studies were carried out to verify the range of equipment plugged into each monitor with only those participants with a full profile (monitoring at least the screen and the computer at the location) taken forward in the study. Where the baseline period had identified unusual energy use profiles, locations and equipment were further investigated to ensure the equipment in use was comparable across the study. Those found to be using older computers and less efficient cathode ray tube screens (although small in number) were excluded from the final study. The power ratings and efficiency of individual pieces of desktop equipment was not explored directly as the study focused on the change in user behaviour and their interaction with the equipment. This does mean that differences in the efficiency of equipment used between groups may place initial energy use at different levels, hence overall findings are presented as percentage savings from baselines as opposed to direct energy use comparisons. The field studies also allowed the research team to identify where additional small power items (such as mobile phone chargers $(21.1 \%)$, desktop fans $(12.2 \%)$ and desk heaters $(5.5 \%))$ where present which was found to be similar across all groups. 
Following this, participants were divided into three groups between the two locations (site $A$ and $B$ ) for the provision of feedback and information, this feedback period lasted a similar duration to the baseline period (100 Days). Site A contained a single group on a single floor of the building and site B contained two groups on separate floors of the same building. Before and after occupant surveys were also conducted along with the provision of energy saving advice before and during the feedback period. The approach to groupings ensured that there were situational similarities between groups, which has been shown to be of importance when leveraging social norms (as noted by Goldstein, Cialdini \& Griskevicius (2008). As the research was based in the field, potential for alternative grouping options were limited. It was noted that those in the 'individual and basic group feedback' set started with lower overall energy use than the other groups (there were variations between all three groups) and this was taken into account in the analysis that follows.

\section{Participants:}

Across all groups the mean age of participants was 45 , with a range of 27 to 63 . There was a gender ratio of 44 to $56 \%$ male to female. Participants were provided with guidance on the process and purpose of the study and the opportunity to opt in or out. The participants can be said to be a combination of individual and group process workers as defined by Laing et al. (cited in Haynes 2008), working in what can be described as 'large open-plan' offices as defined by Bodin Danielsson and Bodin (2008).

Although 90 desks were originally included in the study, due to internal reconfigurations and staff turnover (and exclusions associated with inconsistencies found in the data) the number of participants reduced to 39. Larger sets of group level data were available, however to improve reliability only those individuals that could be followed throughout the study were included. As previously noted, the reduction in the number of participants was a factor of the longer baseline period that intended to improve the reliability of the overall data set and to ensure that short term variation in energy use was not misinterpreted as energy savings. The remaining participants can be said to be representative 
of the wider group in terms of job function, gender and age, with an even distribution across groups. The large reduction in the number of participants must be noted as a limitation of the study and the findings are presented in that context.

\section{Feedback}

Following the baseline period feedback was provided in the form of a visual chart for easy interpretation emailed to participants (see Figures $\mathbf{2}$ and $\mathbf{3}$ ), in each case feedback was administered every two weeks. A positive (smiley/happy) face was used to indicate savings in line with the prescribed goals and a negative (unhappy) face indicated performance not as desired. The day-night breakdown was included to show wastage associated with leaving equipment on overnight.

Figure 2: Basic Group Feedback

Members of the first 'basic feedback group' ( $N=16)$ (located at site A) were provided with basic group level feedback with a goal reduction of $20 \%$, no breakdown of day and night energy consumption and information both on the previous and current averages. See Figure $\mathbf{2}$ for an example of the feedback provided. 
Members of the 'detailed comparative feedback group' $(\mathrm{N}=12)$ (located at site $\mathrm{B}$ ) were provided with detailed group level comparative feedback (comparing performance to another group) with a goal reduction of $20 \%$, a breakdown of day and night energy consumption and information both on the previous and current averages.

Members of 'individual and basic group feedback' set $(\mathrm{N}=11)$ (located at site $\mathrm{B}$ ) were provided with individual feedback detailing personal consumption along with a goal reduction of $20 \%$ and information about their groups performance, highlighting the difference between group performance and their own individual performance, again current and historic feedback was provided. See Figure 3 for an example of the feedback provided.

Figure 3: Group \& Individual Feedback

\section{Education/Information}

Prior to the first feedback point information detailing the importance of saving energy (social norms/attitude) and how to save energy specifically within the workplace (perceived behavioural control) was given to each participant electronically, this included guidance about unplugging unused chargers etc., powering down computers and turning off screens both at the end of the working day 
and during the day when away from the desk (30 and 120 minutes). As the study progressed this guidance was reinforced along with the provision of feedback.

Survey

A range of psycho-social factors were also assessed following the baseline monitoring period and prior to the interventions through an online survey. The survey was divided into questions covering a number of broad themes related to the theories previously reviewed namely, pro-environmental attitude (including issues related to sustainability at home and in the workplace), habit/intention (including issues related to powering down equipment during the day and overnight, use of small electronic devices and turning lights off during the day etc.) and social norms (feelings of pressure to behave a certain way and guilt when not done). Questions were administered on a five point Likert scale ranging from 'strongly disagree' to 'strongly agree' allowing for the generation of Likert scale data. The survey was repeated at the end of the intervention period to assess the impact the interventions made. A number of addition questions were added to the follow up survey to gauge participant engagement and also to judge levels of perceived behavioural control (in terms of how easy or difficult it is to save energy in the workplace). 37 participants completed the survey administered before the interventions period and this reduced to 22 for the survey administered at the end of the intervention period. Due to the level of reduction in the number of participants in the survey carried out after the interventions, the results (of the follow up survey) must be viewed with a note of caution.

\section{Approach to Statistical Analysis}

Energy use and standard deviations were calculated for each group both for the baseline and for the feedback period. Statistical analysis of the energy data was carried out in a number of steps. First, across all groups energy use at the baseline and at each feedback point was considered using a repeated measures Analysis of Variance (ANOVA). Where significant variation was identified $(p=<0.05)$ post-hoc Bonferroni corrected $(p=.00714) t$-tests were conducted to identify where the significance 
occurred. To understand the variation in savings between groups a further repeated measures ANOVA was carried out.

For the before and after surveys a test of internal consistency was carried out using Cronbach's Alpha, which is used to determine if multiple questions in a questionnaire related to the same variable, for instance social norms, reliably measure that variable. In this case it was applied to questions relating to social norms, perceived behavioural control and pro-environmental attitude, with an $\alpha$ is in excess of 0.7 found in each case, thus indicating a good level of internal consistency.

Before and after survey results were considered using paired t-tests across all groups. These were supported by a repeated measures ANOVA to consider differences between the three groups, again where significant differences were identified $(P=<0.05)$ (from the ANOVA) these were followed up by post-hoc Bonferroni corrected ( $\mathrm{p}=.0167) \mathrm{t}$-tests. Further Pearson correlation tests were carried out to understand the relationship between a number of key elements considered in the survey (age and baseline energy and energy savings, pro-environmental attitude and energy savings, perceived behavioural control and energy savings, and feelings of guilt (social normative influence) and energy savings).

\section{Results}

\section{Measured Energy Savings}

For the 'basic feedback group' (office location A) the pre-intervention mean (and therefore baseline) was 303 watt-hours with a standard deviation of 125 watt-hours, for the 'detailed comparative feedback group' (office location B) the mean was 343 watt-hours with a standard deviation of 128 watt-hours and for the 'individual and basic group feedback' set (office location B) the mean was 284 watt-hours with a standard deviation of 118 watt-hours. 
The 'basic feedback group' (location A) achieved energy savings of 18\% (SD=94 watt-hours), 'detailed comparative feedback group' (location B) 28\% (SD=118 watt-hours) and 'individual and basic group feedback' (location $\mathrm{B})$ 10\% (SD=115 watt-hours) with an overall reduction in range and standard deviations (see Figure 4). Those who across all groups started with a baseline energy consumption in excess of the overall mean saved on average $28.6 \%$, conversely those whose consumption was below the mean of the initial baseline saved on average just $9 \%$. Across all groups it was found that overall energy savings were significant $(F(7,288)=2.58, p=.0134)$ and that these significant differences occurred at feedback points 1 ( $9 \%$ reduction from baseline) (t $(72)=3.15, p=.002), 4$ ( $12 \%$ reduction from baseline) $(t(72)=3.04, p=.003)$ and point $6(21 \%$ reduction from baseline) $(t(72)=4.14, p=<.001)$. Although as can be seen in Figure $\mathbf{5}$ there would appear to be an early move towards an increase in energy use (around intervention point 2) which is particularly noticeable for the 'detailed comparative feedback group' and 'individual and basic group feedback' sets, this was found not to be significant ( $5 \%$ increase from baseline) $(t(72)=1.55, p=.06)$. Follow up analysis found no significant difference in the energy savings made between groups during the feedback period $(F(2,34)=1.03, p=.365)$.



Int 1: Basic Group Feedback Int 2: Detailed Group Comparative Feedback Int 3: Basic Group \& Individual Feedback

Figure 4: Box Plots before $\&$ after interventions 


\section{Behavioural Questionnaires}

Participant surveys found no significant differences across all groups combined between the before and after surveys in terms of pro-environmental views $(t(22)=1.35, p=.094)$ and feelings of guilt $(t(22)=0.98, p=.167)$, however a marginally significant improvement in overall self-reported proenvironmental behaviour (which focused on measures around energy use in the office, such as power down rates etc.) was identified $(t(22)=1.74, p=.047)$. Between groups no significant difference in selfreported behaviour was found $(F(2,17)=1.83, p=.189)$.

The follow up survey contained a number of questions that were not included in the pre-intervention survey (including the provision of overall feedback to the research team). Between groups a significant difference was found in terms of perceived behavioural control $(F(2,18)=8.26, p=<.01)$ with these differences being found to occur between the 'basic group feedback' (mean 3.63) and 'individual and basic group feedback' (mean 2) $(t(13)=3.94, p=<.01)$ and between 'detailed comparative group feedback' (mean 2.66) and 'individual and basic group feedback' $(t(15)=2.63, p=<.01)$. This suggests that those who received individual feedback (along with feedback on their own group) felt they had less control than those who received just group level feedback or comparative group level feedback. The reason for this is not immediately clear, however, the individual feedback group did save the least amount of energy ( $10 \%$ versus $18 \%$ and $28 \%$ for the other groups) which may be influential, as may the stronger feelings of group membership in the other groups with individuals alone feeling they have little influence.

\section{Combined Energy Saving and Questionnaire Results}

Combining the observed energy savings and questionnaire responses this study found no significant correlation between pro-environmental attitude and energy savings $(r=0.29, p=0.86)$, perceived behavioural control and energy savings $(r=0.52, p=0.98)$, energy savings and feelings of guilt (social normative influence) $(r=0.01, p=0.5)$ or energy savings and age $(r=0.46, p=0.96)$. 


\section{Discussion}

This study demonstrated that significant energy savings are possible in the commercial office environment through the use of feedback, goal setting and education/ information. The savings achieved overall were in line with the findings of Darby (2006) who suggested $20 \%$ saving should be possible. Not unexpectedly those who started with a baseline energy consumption above the overall mean (and therefore with greatest potential for energy savings) saved the most energy. If the highest energy users across all groups in the study could reduce energy consumption to the mean $38 \%$ energy savings could be realised, furthermore if those at the mean level could reduce to the minimum (which is technically possible, although in practical terms may be unlikely) savings of up to $41 \%$ could be realised, thus savings of $38-41 \%$ should be theoretically possible. This is comparable to the findings of Murtagh et al. (2013) who noted possible savings of up to $32 \%$ in a university context.

\section{Pattern of Energy Savings}

Dwyer et al. (1993) notes that in the residential setting removing feedback results in a quick reduction in energy savings. However, studies in commercial settings (Murtagh et al., 2013 and Gulbinas \& Taylor 2014) note that the impact of feedback reduces more gradually while at the same time energy savings may take longer to take hold than the residential setting and as such are less 'elastic' than the residential sector (Murtagh et al., 2013). This is however in contrast to the findings of Wakiyama et al. (2014), who found that during an exogenous shock (in that case the Fukushima nuclear crises) households responded more gradually than large electricity users (but the reductions were more likely to persist). The present study reinforces the findings of Murtagh et al. (2013) and Gulbinas and Taylor (2014) as, despite an initially significant reduction in energy (feedback point 1), consistent and significant energy savings did not occur until later in the study (feedback point $4 \& 6$ ). This finding also highlights the importance of the length of the monitoring (and baseline) period as shorter periods may have pointed to a different outcome (in terms of elasticity). Furthermore in the residential sector it has been observed that notable energy reduction can be observed in the two to three days following 
the provision of feedback (Peschiera, Taylor \& Siegel, 2010), however in this study no significant energy reduction was noted in that timeframe. As such the impact of feedback in the commercial sector would appear to function differently to that of the residential sector, the elasticity of the change in each sector may however be influenced by the immediacy of the driving force behind any reduction (such as an exogenous shock). For each group when 66 days of feedback (between feedback points 4 and 5) has passed, which is as noted by Lally et al. (2010) the median time taken for the 'automaticity' (as defined by Bargh, 1994, in Chatterton, 2011) of an action to peak, a downward trend in energy consumption emerges suggesting that feedback may be beginning to form a habit. However, as can be seen from the statistics and Figure $\mathbf{5}$ this downward trend does not stay persistent and there remains a question over how long lasting the impact of feedback may be. As noted by Darby et al. (2016) a degree of behavioural reinforcement may still be required. 


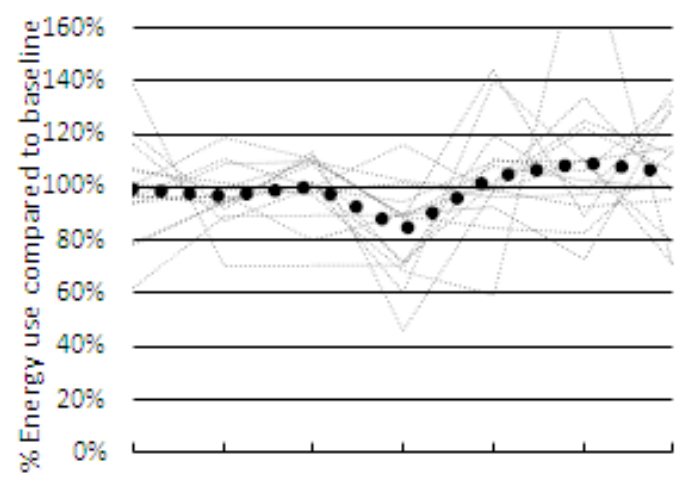

Time - Baseline Period 100 Days

Int 1 - Before

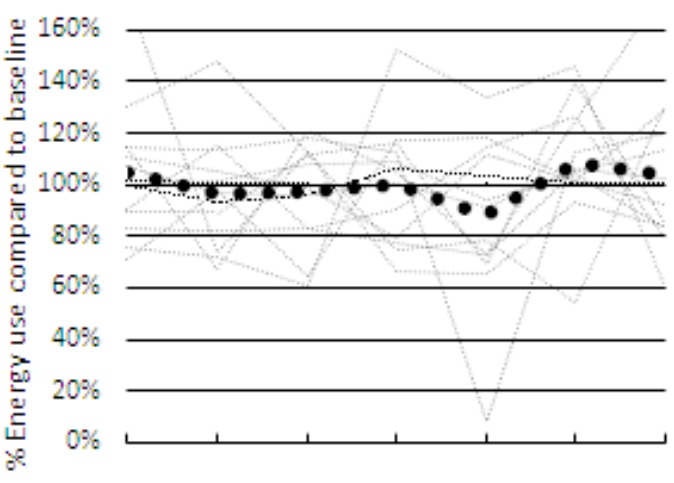

Time - Baseline Period 100 Days

Int 2 - Before

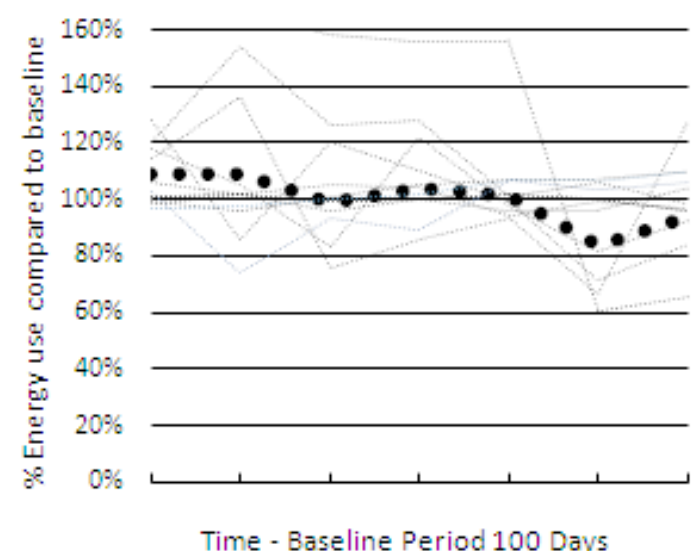

Int 3 - Before

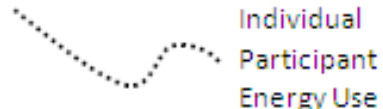

Energy Use



Int 1 - After

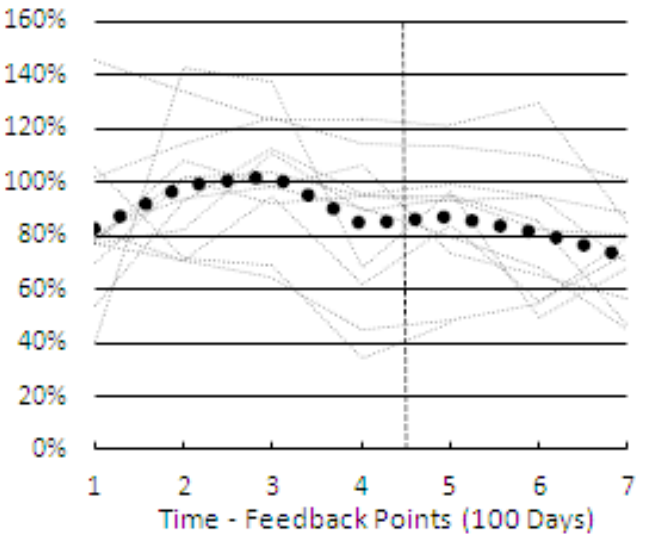

Int 2 - After

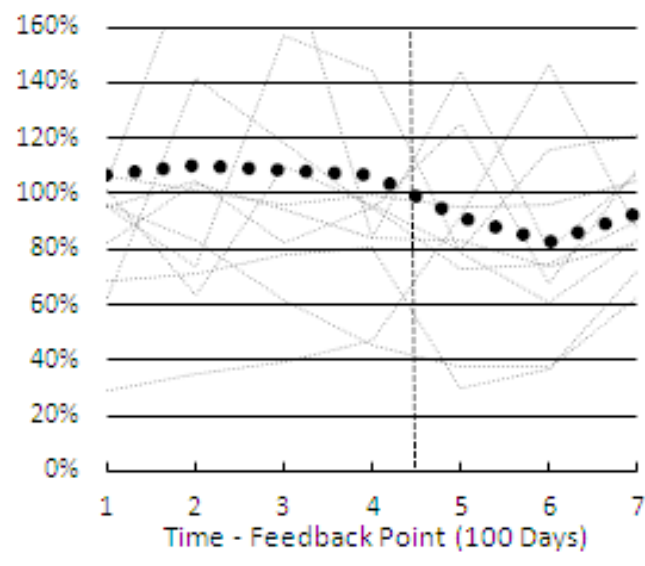

Int 3 - After

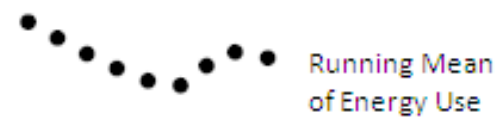

Int 1: Basic Group Feedback - Int2: Detailed Group Comparative Feedback Int 3: Basic Group \& Individal Feedback

Figure 5: Energy use patterns 


\section{Changes in Attitude, Perceived Behavioural Control and Social Norms}

As noted, the before and after surveys found no significant difference across all groups in terms of pro-environmental views (attitude) and feelings of guilt (social norms), however a significant difference was found in terms of self-reported pro-environmental behaviour. This indicates that change in habit may have occurred without a change in attitude and aligns well with other studies in the non-domestic setting (Siero et al., 1996 \& Tetlow, van Dronkelaar, Beaman, Elmualim \& Couling, 2015). This can be highlighted as a key difference to the domestic sector where pro-environmental attitude is seen as a key factor of energy saving (see Abrahamse et al., 2005) and would appear to contradict Francis et al. (2004) who noted a strong link between attitude and intention, and behaviour. However, as noted by Chatterton (2011) this may be related to the 'value-action gap' where stated values do not necessarily correspond to action. This change in habit without an underlying change in attitude may also be a factor of a range of issues encountered in the workplace that could influence behaviour and are not commonly encountered in residential settings (culture, organisational focus and structure (Tudor et al., 2008) and jobs satisfaction (Brent and Freathy, 1997)).

Although initially this research would seem to support the theory that comparative feedback among groups is more successful than individual feedback (for example Siero et al., 1996 and Gulbinas \& Taylor, 2014) the observed difference was not statistically significant in this case. This perhaps suggests that the links made to group membership and competition (Wit \& Wilke, 1988) may not be as strong as the theory suggests when applied in the commercial office environment.

However, as noted by Abrahamse et al. (2007) large in group variation combined with the smaller number of participants within each group ('basic feedback group' $\mathrm{N}=16$, 'detailed comparative feedback group' $\mathrm{N}=12$, 'individual and basic group feedback' $\mathrm{N}=11$ ) may be a contributory factor to this, making it difficult to find significance in variation between groups and therefore intervention types, thus this result should be considered in that context. It is worth noting however, that those in 
the 'individual and basic group feedback' set started with lower overall energy use than the other groups, suggesting less potential for energy savings. It could be argued that this supports the idea that group membership was less influential. As noted below however, group membership may impact on perceived behavioural control which may, in some cases, also impact upon energy saving behaviour. As such the benefits of group membership may need further exploration in studies with long term baseline and intervention periods.

The importance of perceived behavioural control in the commercial sector has been highlighted by previous authors (for example Menzes et al., 2012 \& Tetlow et al., 2013). Although a difference between groups was found in terms of perceived behavioural control (with the 'individual and group feedback' set having the lowest level of perceived behavioural control), no correlation was found between levels of perceived behavioural control and realised energy savings. As the level of control available across all three groups are similar it is not immediately clear why perceived behavioural control would be lower in one group, it could be related to a realisation that the levels of energy savings achieved are below target (this was the case for the 'individual and group feedback' set) and a need to put forward a reason due to social normative influence. It may be that those who received group level or group comparative feedback (as opposed to individual feedback) have a stronger sense of group membership which increases levels of perceived behavioural control, compared to individuals who feel they can have little influence. Furthermore, no correlation was found between pro-environmental attitude or feelings of guilt (social norms) and energy savings. This further supports theory that in the commercial sector habit may be changed without changing attitude through the provision of feedback and information.

Implications

For the building manager, although the methods used here resulted in significant energy savings, as underlying environmental attitudes have not changed it may be that a less individualistic approach 
where externalities (to the individual) such as workplace culture and practice are taken into account, could be of benefit. Campaigns to reduce workplace energy use may be more successful if feedback and goal setting were combined with a management campaign highlighting the accepted workplace culture (or expected workplace norm) and practice. In this context, energy saving campaigns in the workplace may not need to target the underlying environmental attitude of occupants (which aligns with the findings of Siero et al., 1996), with accepted workplace culture (or norms) instead used to influence workplace behaviour.

Changes to such workplace culture and practice may contribute to 'facilitating conditions' as an 'external enabler'. As perceived behavioural control, other than desk level control, in the commercial office may be largely invisible, it may be that focusing on workplace practice and culture can also be used in conjunction with feedback, through an active approach to workplace management, to reconnect occupants to the space they occupy enhancing the wider 'facilitating conditions'. Figure 6 presents an outline of such a model (and is a modified version of Figure 1).

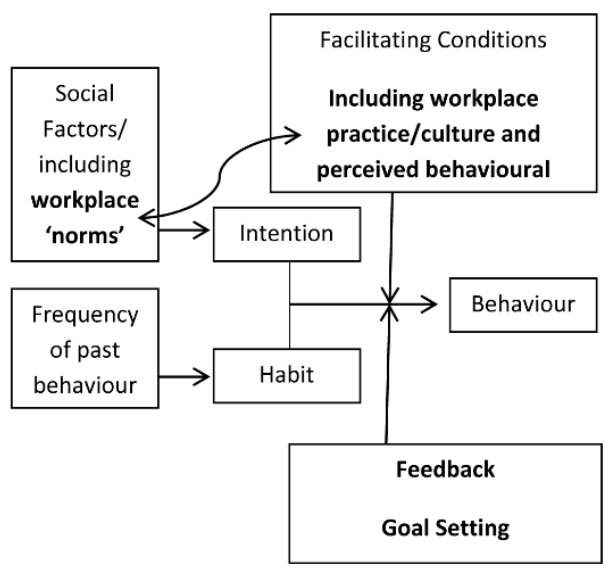

Figure 6: Revised version of Triandis" "Theory of Interpersonal Behaviour", adapted from Chatterton, 
As noted by Haynes (2008) and supported by Mulville, Callaghan and Isaac (in press) an active approach to workplace management supporting the needs of the user and the organisation may be of benefit in understanding occupant satisfaction and encouraging preferred behaviours. Darby et al. (2016) suggests that providing users with the means to understand the impacts of their own actions could help to change the energy culture in the building, making control move visible while increasing personal responsibility. In practical terms an active approach to workplace management could include monitoring and feedback in a continuous closed loop system (as suggested by Darby et al., 2016) which could take advantage of the capability of information technology systems, building automation and more innovative facilities management systems. The feedback required could be provided electronically with opportunities for building users to in turn engage with the feedback. Such an approach could align with the observed growth in the use of 'performance leases' (Janda et al., 2016) and offer the potential for enhanced facilities management services which could be combined with efforts to improve workplace health, wellbeing and productivity. Where feedback is coupled with education and information regarding user controls, such approaches could help to reduce the building performance gap, which has been linked to the influenced occupant behaviour (van Dronkelaar et al., 2016).

More widely, in the context of the European Commission's ambitious plans to improve energy efficiency and given the growing recognition of the influence of occupant behaviour on energy use (Darby et al., 2016), this is an area likely to come into increasing focus. At a policy level incentive schemes to encourage businesses to engage in such behaviour change campaigns, embedded in active approaches to workplace management, could offer improvements in efficiency and reductions in emissions at relatively low costs (in comparison to interventions association with the building fabric, systems and renewables). Arguably, such approaches could help to address some of the challenges presented by the existing stock and in turn offer benefits beyond the workplace. 


\section{Conclusions}

The study demonstrated that, in the commercial office setting, it is possible to save energy through behaviour change by utilising the provision of feedback, goal setting and information. The paper also suggests that it may be possible to achieve these savings without a corresponding change to proenvironmental attitude or perceived social norms. On average the study found savings of $18.8 \%$ to be possible, with savings of $28 \%$ for the comparative feedback group, $18 \%$ for the basic feedback group and $10 \%$ for the individual and basic feedback group. The impact of group membership and perceived behavioural control were in this case found not to be as significant as previously expected but may still contribute to the wider facilitating conditions.

For the building manager, feedback and goal setting may help improve workplace behaviour in relation to energy consumption. Within this a focus on workplace culture and practice may be of greater importance than focusing on the underlying environmental attitude of occupants. This suggests that a less passive and individualistic approach by senior management to behaviour change may be of benefit in such campaigns in the workplace. Arguably, such an approach may improve levels of perceived behavioural control, thus harnessing the potential benefits. In practical terms an active approach to workplace management with a continuous feedback loop may be of benefit.

As this study focused on a single business, it was not possible to test the above suggestions across sectors and this is an area for further research, along with consideration of how job function has an impact on energy use behaviour. However, the potential energy savings suggested do, in broad terms, align well with other studies in the field (such as Darby et al. (2016) and Gulbinas \& Taylor (2014)), suggesting the findings could be generalised to other situations. At the scale of an individual building the savings noted may be relatively small, however if such savings could be scaled up across multiple built assets or at a national or regional scale the cost savings and emission reductions may be significant. 
The findings of this paper need to be considered in the context of a number of limitations. The original study began with 90 desk locations between two locations with three groups, however due to internal reconfigurations and staff turnover (and exclusions associated with identified inconsistencies in the data) it was possible to follow only 39 of these individuals through to the completion of the study. In addition not all participants completed the before and after survey, with 37 completing the before survey and 22 the after survey and again this has an impact on the findings. $95 \%$ of those who completed the survey at the end of the study indicated that they did engage with the feedback provided regularly. This suggests that those who did participate in the follow up survey were perhaps more engaged with the overall study than the wider group. The format feedback was delivered in did not make it possible to confirm this (engagement with feedback) with empirical data and, as such, this is self-reported engagement which may be unreliable. If those who did not complete the follow up survey did not engage with feedback, the overall level of engagement drops to $56.7 \%$, which is similar to the findings of Murtagh et al. (2013).

\section{References}

Abrahamse, W., Steg, L., Charles, V. \& Rothengatter, T. (2005). A review of intervention studies aimed at household energy conservation. Journal of Environmental Psychology, 25, 273-291. doi:10.1016/j.jenvp.2005.08.002

Abrahamse, W., Steg, L., Charles, V. \& Rothengatter, T. (2007). The effect of tailored information, goal setting, and tailored feedback on household energy use, energy-related behaviours, and behavioural antecedents. Journal of Environmental Psychology, 27, 256-276. doi: 10.1016/i.jenvp.2007.08.002

Ajzen, I. (1991). The theory of planned behaviour. Organisational Behaviour and Human Decision Processes, 50, 179-211. doi:10.1016/0749-5978(91)90020-T

Benders, R., Kok, R., Moll, H., Wiersma, G. \& Noorman, K. (2006). New approaches for household energy conservation: In search of personal household energy budgets and energy reduction options. Energy Policy, 34, 3612-3622. doi:10.1016/j.enpol.2005.08.005

Bodin Danielsson, C. \& Bodin, L. (2008). Office-type in relation to health, well-being and job satisfaction among employees. Environment \& Behaviour, 40, 636-668. doi:10.1177/0013916507307459 
Bostrom, A. \& Fischhoff, B. (2001). Communicating health risks of global climate change. Research in Social Problems and Public Policy, 9, 31-56. doi: 10.1016/S0196-1152(01)80023-5

Brent, R. \& Freathy, P. (1997). Motivating the employee in the independent retail sector. Journal of Retailing and Consumer Services, 43, 201-208. doi:10.1016/S0969-6989(96)00045-8

Building Performance Institute Europe (2011). Europe's buildings under the microscope. A country by country review of the energy performance of buildings. Belgium: Building Performance Institute Europe. Retrieved from bpie.eu: http://bpie.eu/publication/europes-buildings-under-the$\underline{\text { microscope/ }}$

Bull, R., Lemon, M., Fleming, P., Stuart G. and Everitt, D. (2014). Digitally Engaging and Empowering Employees for Energy Demand Reduction: A New Approach for the Next Generation? Paper presented at American Council for an Energy Efficient Economy Conference 2014, Asilomar. Retrieved from acee.org: http://aceee.org/files/proceedings/2014/data/papers/7-99.pdf

Carrico, A. \& Riemer, M. (2011). Motivating energy conservation in the workplace: An evaluation of the use of group-level feedback and peer education. Journal of Environmental Psychology, 31, 1-13. doi:10.1016/i.jenvp.2010.11.004

Chen, J. \& Ahn, C. (2014). Assessing occupants' energy load variation through existing wireless network infrastructure in commercial and educational buildings. Energy and Buildings, 82,540-549. doi:10.1016/j.enbuild.2014.07.053

Chatterton, T. (2011). An introduction to thinking about 'Energy Behaviour': A multi-model approach. Retrieved from gov.co.uk:

https://www.gov.uk/government/uploads/system/uploads/attachment data/file/48256/3887-introthinking-energy-behaviours.pdf

Cialdini, R.B., Kallgren, C.A. \& Reno, R.R. (1991). A focus theory of normative conduct: A theoretical refinement and re-evaluation of the role of norms in human behaviour. Advances in Experimental Psychology, 24, 200-234. doi:10.1016/S0065-2601(08)60330-5

Dakin, S. \& Arrowood, A. (1981). The social comparison of ability. Human Relations, 34, 89-109. doi:10.1177/001872678103400201

Darby, H., Elmualim, A., Clements-Croome, D., Yearley, T. \& Box, W. (2016). Influence of occupants' behaviour on energy and carbon emission reduction in a higher education building in the UK. Intelligent Buildings International. doi:10.1080/17508975.2016.1139535

Darby, S. (2006). The effectiveness of feedback on energy consumption. A review for DEFRA of the literature on metering, billing and direct displays. University of Oxford: Environmental Change Institute.

De Young, R., Boerschig S., Carney, S. Dillenbeck, A., Elster, M., Horst, S., Kleiner, B. \& Thomson, B. (1995). Recycling in multi-family dwellings: Increasing participation and decreasing contamination. Population and Environment: A Journal of Interdisciplinary Studies, 16, 253-267. doi:10.1007/BF02331920 
Dwyer, W., Leeming, F., Cobern, M, Porter, B. \& Jackson, J. (1993). Critical review of behaviour interventions to preserve the environment: Research since 1980. Environment and Behaviour, 25, 275-321. doi:10.1177/0013916593255001

The European Commission (2014). The 2030 climate and energy framework. Retrieved from the European Commission: http://ec.europa.eu/clima/policies/strategies/2030/index en.htm

Francis, J., Eccles, M. P., Johnston, M., Walker, A. E., Grimshaw, J. M., Foy, R., Kaner, E. F. S., Smith, L. \& Bonetti, D. (2004). Constructing questionnaires based on the theory of planned behaviour: A manual for health services researchers. Newcastle upon Tyne: Centre for Health Services Research. Retrieved from openaccess.city.ac.uk: http://openaccess.city.ac.uk/1735/1/TPB\%20Manual\%20FINAL\%20May2004.pdf

Gilbert, T., Fiske, S. \& Lindzey, G. (1998). Handbook of social psychology (4th edition) (pp. 680-740). New York: McGraw-Hill.

Goldstein, N.J., Cialdini, R.B. \& Griskevicius, V. (2008). A room with a viewpoint: Using social norms to motivate environmental conservation in hotels. Journal of Consumer Research, 35, 472-482. doi:10.1086/586910

Gulbinas, R \& Taylor, J. (2014). Effects of real-time eco-feedback and organizational network dynamics on energy efficient behaviour in commercial buildings. Energy and Buildings, 84, 493-500. doi:10.1016/j.enbuild.2014.08.017

Haynes, B. (2008). The impact of office comfort on productivity. Journal of Facilities Management, 6 , 37-51. doi: $\underline{10.1108 / 14725960810847459}$

Hopper, J. \& Nielsen, J. (1991). Recycling as altruistic behaviour: normative and behavioural strategies to expand participation in a community recycling program. Environment and Behaviour, 23, 195-220. doi:10.1177/0013916591232004

Jackson, S. \& Zedeck, S. (1982). Explaining performance variability: Contribution of goal setting, task characteristics, and evaluative contexts. Journal of Applied Psychology, 67. 59-768.

doi.org/10.1037/0021-9010.67.6.759

Janda, K.B., Bright, S., Patrick, J., Wilkinson, S. and Dixon, T.J. (2016). The evolution of green leases: Towards inter-organisational environmental governance. Building Research and Information, 44, 660-674. doi:10.1080/09613218.2016.1142811

Jenkins, D. P., Singh, H., \& Eames, P. C. (2009). Interventions for large-scale carbon emission reduction in future UK offices. Energy and Buildings, 41, 1374-1380.

doi:10.1016/j.enbuild.2009.08.002

Junnila, S. (2007). The potential effect of end-users on energy conservation in office buildings. Facilities, 25, 329-339. doi:10.1108/02632770710753352

Kawamoto, K., Shimoda, Y., \& Mizuno, M. (2003). Energy saving potential of office equipment power management. Energy and Buildings, 36, 915-923. doi:10.1016/j.enbuild.2004.02.004 
Katzev, R. \& Mishima, H. (1992). The use of posted feedback to promote recycling. Psychological Reports, 71, 259-264. doi:10.2466/pro.1992.71.1.259

Lally, P., van Jaarsveld, C.H.M., Potts, H.W.W. \& Wardle, J. (2010). How are habits formed: Modelling habit formation in the real world. European Journal of Social Psychology, 40, 998-1009. doi: $\underline{10.1002 / \text { ejsp.674 }}$

Maréchal, K. (2010). Not irrational but habitual: The importance of "behavioural lock-in" in energy consumption. Ecological Economics, 69, 1104-1114. doi:10.1016/i.ecolecon.2009.12.004

Menzes, A.C., Tetlow, R., Beaman, C.P., Cripps, A., Bouchlaghem, D. \& Buswell, R. (2012). Assessing the impact of occupant behaviour on the electricity consumption for lighting and small power in office buildings. Paper presented at the $7^{\text {th }}$ International Conference on Innovation in Architecture, Engineering \& Construction, Sao Paolo, Brazil.

Miller, D. \& Prentice, D. (1996). The construction of social norms and standards. In Kruglanski, A.W. Higgins, E.T (Eds.). (1996). Social psychology: handbook of basic principles (pp. 799-829). New York: Guilford Press.

Mulville, M., Callaghan, N. \& Isaac, D. (in press). The impact of the ambient environment and building configuration on occupant productivity in open-plan commercial offices. Journal of Corporate Real Estate.

Mulville, M., Jones, K. \& Huebner, G. (2014). The potential for energy reduction in UK commercial offices through effective management and behaviour change. Architectural Engineering and Design Management, 10, 79-90. doi:10.1080/17452007.2013.837250

Murtagh, N., Nati, M., Hadley, W.R., Gatersleben, B., Gluhak, A., Ali Imran, M. \& Uzzell, D. (2013). Individual energy use and feedback in an office setting: A field trial. Energy Policy, 62, 717-728. doi:10.1016/i.enpol.2013.07.090

Nilsson, A., Andersson, K. \& Bergstad, C.J. (2015). Energy behaviours at the office: An intervention study of the use of equipment. Applied Energy, 146, 434-441. doi:10.1016/j.apenergy.2015.02.045

Nolan, J., Schultz, P., Cialdini, R., Goldstien, N. \& Griskevicius, V. (2008). Normative social influence is under-detected. Personality and Social Psychology Bulletin, 34, 913-923.

doi:10.1177/0146167208316691

Perkins, H., Haines, M. \& Rice, R. 2005. Misperceiving the College Drinking Norm and Related Problems: A Nationwide Study of Exposure to Prevention Information, Perceived Norms, and Student Alcohol Misuse. Journal of Studies on Alcohol, 66, 470-478. doi: 10.15288/isa.2005.66.470

Peschiera, G., Taylor, J.E. \& Siegel J.A. (2010). Response-relapse patterns of building occupant electricity consumption following exposure to personal, contextualized and occupant peer network utilization data. Energy and Buildings, 42, 1329-1336. doi:10.1016/i.enbuild.2010.03.001

Scherbaum, C., Popovich, P. \& Finnlinson, S. (2008). Exploring individual-level factors related to employee energy-conservation behaviours at work. Journal of Applied Social Psychology, 38, 818835. doi:10.1111/j.1559-1816.2007.00328.x 
Schultz, P., Nolan, J., Cialdini, R., Goldstein, N. \& Griskevicius, V. (2007). The constructive, destructive and reconstructive power of social norms. Journal of Psychological Science, 18, 429-434. Retrieved from https://www.jsmf.org/meetings/2008/july/social\%20norms\%20Cialdini.pdf

Schultz, P., Oskamp, S. \& Mainieri, T. (1995). Who recycles and when? A review of personal and situational factors. Journal of Environmental Psychology, 15, 105-121. Retrieved from http://americarecyclesday.org/wp-content/uploads/2013/07/Schultz-Oskamp-1995.pdf

Seligman, G., Becker, L. \& Darley, J. (1981). Encouraging residential energy conservation through feedback. In Baum, A. \& Singer J. (Eds.). (1981). Advances in Environmental Psychology, Energy conservation: Psychological Perspectives (Vol. 3). New Jersey: Erlbaum Associates.

Shalley, C., Oldham, G. \& Porac, J. (1987). Effects of goal difficulty, goal setting method, and expected external evaluation on intrinsic motivation. Academy of Management Journal, 30, 553-563. doi:10.2307/256118

Siero, F., Bakkerm A., Dekker, G. \& van Der Burg, M. (1996). Changing organisational energy consumption behaviour through comparative feedback. Journal of Environmental Psychology, 16, 235-246. doi:10.1006/jevp.1996.0019

Siero, S., Boon, M., Kok, G. \& Siero F. (1989). Modification of driving behaviour in a large transportation organisation: A field experiment. Journal of Applied Psychology, 74, 417-423. doi:10.1037/0021-9010.74.3.417

Stern, P., Dietz, T., Abel, T., Guagnano, G., \& Kalof, L. (1999). A value-belief-norm theory of support for social movements: The case of environmental concern. Human Ecology Review, 6, 81-97. Retrieved from http://citeseerx.ist.psu.edu/viewdoc/download?doi=10.1.1.195.5410\&rep=rep1\&type=pdf

Stern, P \& Gardener, G. (1981). Psychological Research and Energy Policy. American Psychologist, 36, 29-342. doi:10.1037/0003-066X.36.4.329

Stern, P. (2000). Toward a coherent theory of environmentally significant behaviour. Journal of Social Issues, 56, 407-424. doi: 10.1111/0022-4537.00175

Tajfel, H. (1978). Social categorisation, social identity and social comparison. In Tajifel, H. \& Turner, J. (Eds.).(1978) Differentiation between social groups: Studies in the social psychology of intergroup relations (pp.61-76). London: Academic Press.

Tetlow, R.M., Beaman, C.P., Elmualim, A.A \& Couling, K. 2013. Targeting automatic behaviour to reduce small power electricity consumption in office buildings. Paper presented at the 4th Annual TSBE Eng. D. Conference, Reading, UK.

Tetlow, R.M, van Dronkelaar, C., Beaman, C.P., Elmualim \& A.A, Couling, K. 2015. Identifying behavioural predictors of small power electricity consumption in office buildings. Building and Environment, 92, 75-85. doi:10.1016/j.buildenv.2015.04.009

Tudor, T., Barr, S. \& Gilg, A. (2008). A novel conceptual framework for examining environmental behaviour change in large organisations: A case study of the Cornwall National Health Service (NHS) in the United Kingdom. Environment and Behaviour, 40, 426-450. doi: 10.1177/0013916507300664 
van Dronkelaar, C., Dowson, M., Spataru, C and Mumovic, D. (2015). A review of the regulatory energy performance gap and its underlying causes in non-domestic buildings. Frontiers in Mechanical Engineering. doi:10.3389/fmech.2015.00017

van Houwelingen, J. \& van Raaij F. (1989). The Effect of Goal-Setting and Daily Electronic Feedback on In-Home Energy Use. Journal of Consumer Research, 16, 98-105. doi:10.1086/209197

van Knippenberg, A., Wilke, H. \& de Vries, N. (1981). Social comparison on two dimensions.

European Journal of Psychology, 11, 267-283. doi: 10.1002/ejsp.2420110303

Wakiyama, T., Zusman, E. \& Monogan III, J.E. (2014). Can a low-carbon-energy transition be sustainable in post Fukushima Japan? Assessing the varying impacts of exogenous shocks. Energy Policy, 73, 654-666. doi:10.1016/j.enpol.2014.06.017

Wechsler, H., Nelson, T., Lee, J., Seiberg, M., Lewis, C., \& Keeling, R. (2003). Perception and reality: A national evaluation of social norms marketing interventions to reduce college students' heavy alcohol use. Quarterly Journal of Studies on Alcohol, 64, 484-494. Retrieved from http://diseaseriskindex.harvard.edu/cas/Documents/social norms/484-Wechsler.pdf

Werch, C., Pappas, D., Carlson, J., DiClemente, C., Chally, P., \& Sinder, J. (2000). Results of a social norm intervention to prevent binge drinking among first-year residential college students. Journal of American College Health, 49, 85-92. doi: 10.1080/07448480009596288

Wit, A. \& Wilke, H. (1992). The effect of social categorisation on cooperation in three types of social dilemmas. Journal of Economic Psychology, 3, 35-151. doi:10.1016/0167-4870(92)90056-D

Zhang, T., Siebers, P. O., \& Aickelin, U. (2011). Modelling electricity consumption in office buildings: An agent based approach. Energy and Buildings, 43, 2882-2892. doi:10.1016/j.enbuild.2011.07.007 